\title{
A POROUS ACTUATOR FOR AN ISFET-BASED COULOMETRIC SENSOR-ACTUATOR SYSTEM
}

\author{
J. Luo, W. Olthuis and P. Bergveld \\ MESA Research Institute, Department of Electrical Engineering, \\ M. Bos and W.E. van der Linden \\ Department of Chemical Technology,
}

University of Twente, P.O. box 217, 7500 AE Enschede, The Netherlands.

\begin{abstract}
$\underline{\text { Abstract }}$
The early developed prototype ISFET-based coulometric sensor-actuator system suffers from delay in response due to the non-zero distance between the sensor and actuator. This paper describes a new configuration of a sensoractuator device which employs a porous noble metal electrode as the actuator closely spaced around and over the gate of the ISFET. The new device was characterized by performing an acid-to-base titration. The results showed that the delay time was considerably reduced. The titration times were found to be from 0.1 to 4.0 seconds. The corresponding concentrations of titrated species were from 0.5 to $15 \mathrm{mM}$.
\end{abstract}

\section{Introduction}

Acid/base titration or chemical micro-environmental control can be achieved by incorporating an actuator closely shaped around the sensor. A typical example is an ISFET-based coulometric sensor-actuator system. If a current is applied to the actuator, the titrants generated by the electrolysis of water will change the $\mathrm{pH}$ in the vicinity of the gate of the ISFET. The output of the ISFET can be monitored either for an acid/base titration or to enable control of the actuating current source by means of a computerized set-up to keep locally the $\mathrm{pH}$ at a desired value. Such systems have been successfully applied to the acid/base titration [1], the construction of a pH-static enzyme sensor [2] and a low-drift carbon dioxide sensor [3]. However, there are some shortcomings with the earlier developed prototype devices. Firstly, the non-zero distance (ca. $20 \mu \mathrm{m}$ ) between the sensor (gate of the ISFET) and the actuator causes a delay in response resulting from the diffusion of the species between the sensor and actuator. Since the delay time is dependent on the concentration of the titrated species and the current density applied [4], it will make the measurement less accurate. Secondly, if a membrane is cast onto the device, e.g., for the construction of a pH-static enzyme sensor, there will be a $\mathrm{pH}$ gradient inside the membrane because of the diffusion of species from the bulk to the actuator. The $\mathrm{pH}$ control inside the membrane is not homogeneous, which somewhat limits the advantage of this kind of sensor. In order to circumvent the above-mentioned problems, a new structure for an ISFET-based sensoractuator device, which employs a porous noble metal as the actuator closely spaced around and over the gate of ISFET, is proposed in this paper.

\section{Fabrication of the device}

Procedures

The procedure to make the device is shown in Fig.1ad. In the first place, the ISFET was made by standard NMOS techniques [5]. The gate dielectric is $\mathrm{SiO}_{2} / \mathrm{Ta}_{2} \mathrm{O}_{5}$. Next, a thin layer of gold $(0.6 \mu \mathrm{m})$ was deposited around the gate area by evaporation (Fig.1a). At this stage, a thin sacrificial layer was applied onto the gate area for protecting the ISFET from damage in the later process. Here, a $1 \mu \mathrm{m}$ sputtered $\mathrm{SiO}_{2} / \mathrm{Si} / \mathrm{SiO}_{2}$ thin film was adopted (Fig.1b). Furthermore, a gold thick-film paste (DuPont 9910) was applied over the evaporated gold layer (Fig.1c). After drying, the paste was sintered at $600{ }^{\circ} \mathrm{C}$ in a nitrogen ambient for one hour. Finally, the sacrificial layer as well as the glassy compound of the thick-film paste were removed by etching. The etching was carried out in three steps. First, the device was immersed in a buffered HF solution for one hour to etch the glassy compound of the thick-film paste and the upper silicon dioxide of the sacrificial layer. The long etching time is necessary for a complete removal of the glassy compound existing in the thick-film paste. Second, the silicon layer was etched by $\mathrm{KOH}$ solution at $73{ }^{\circ} \mathrm{C}$ for 10 minutes. The etching of the final layer of silicon dioxide was proceeded again in a buffered HF solution for 5 minutes. A porous gold layer was then formed closely above the gate (Fig.1d). The thickness of the layer is about 25 to $50 \mu \mathrm{m}$. 

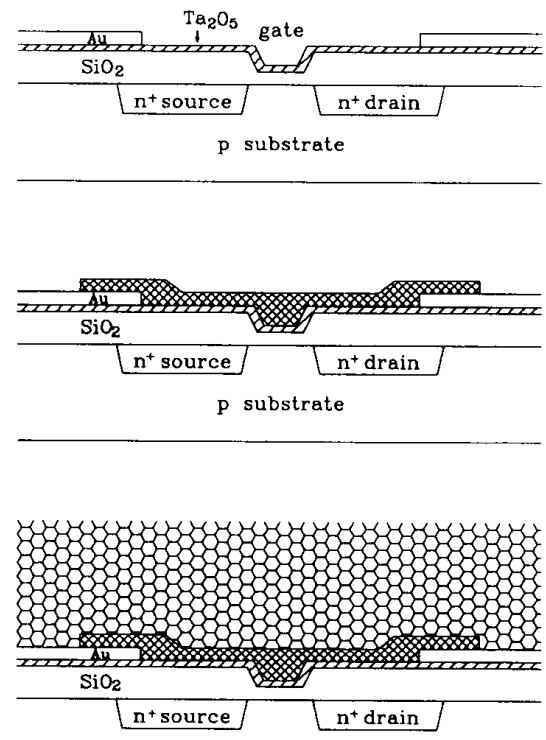

p substrate

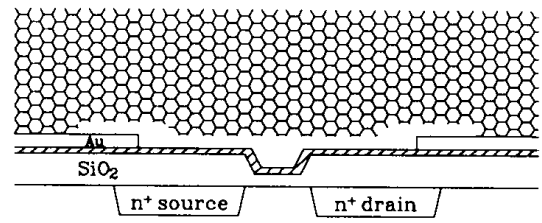

p substrate

Fig.1 (a) ISFET with an evaporated gold layer. (b) Deposition of a sacrificial layer. (c) Applying of a gold thick-film paste. (d) Etching of the glassy compound of the thick-film paste and the sacrificial layer.

\section{The realization of the porous structure}

Since the porous actuator is made from gold thickfilm paste, the parameters in the fabrication are very important and should be optimized. In the particular application of making a porous structure, the parameters in the standard fabrication procedures have to be modified to meet the requirements. With respect to this, no systematic studies have been found dealing with the relation between the porous microstructure and the parameters in the fabrication process. In fact, the most critical parameter is the sintering temperature. The temperature recommended by the company was found to be too high to obtain a porous structure. At this temperature the particles are almost completely melted and fused together. The porous structure, however, only requires the particles to be partly connected, so the corresponding sintering temperature should be lower. The proper sintering tempera- ture for a porous structure depends on the size of the particles. The smaller the particles, the lower the temperature required. For a given thick-film paste, the microstructure of the formed thick film is then welldetermined by the temperature of sintering. The thick-film paste we used in the fabrication of the porous actuator is DuPont 9910 gold paste. The size of the gold particles is found to be $0-3 \mu \mathrm{m}$ in diameter. The microstructure of the thick-film paste after sintering at different temperatures was checked by scanning electron micrography (SEM). Fig.2a-c show the microstructures of the thick-film pastes sintered in nitrogen ambient at 500, 600 and $700{ }^{\circ} \mathrm{C}$,

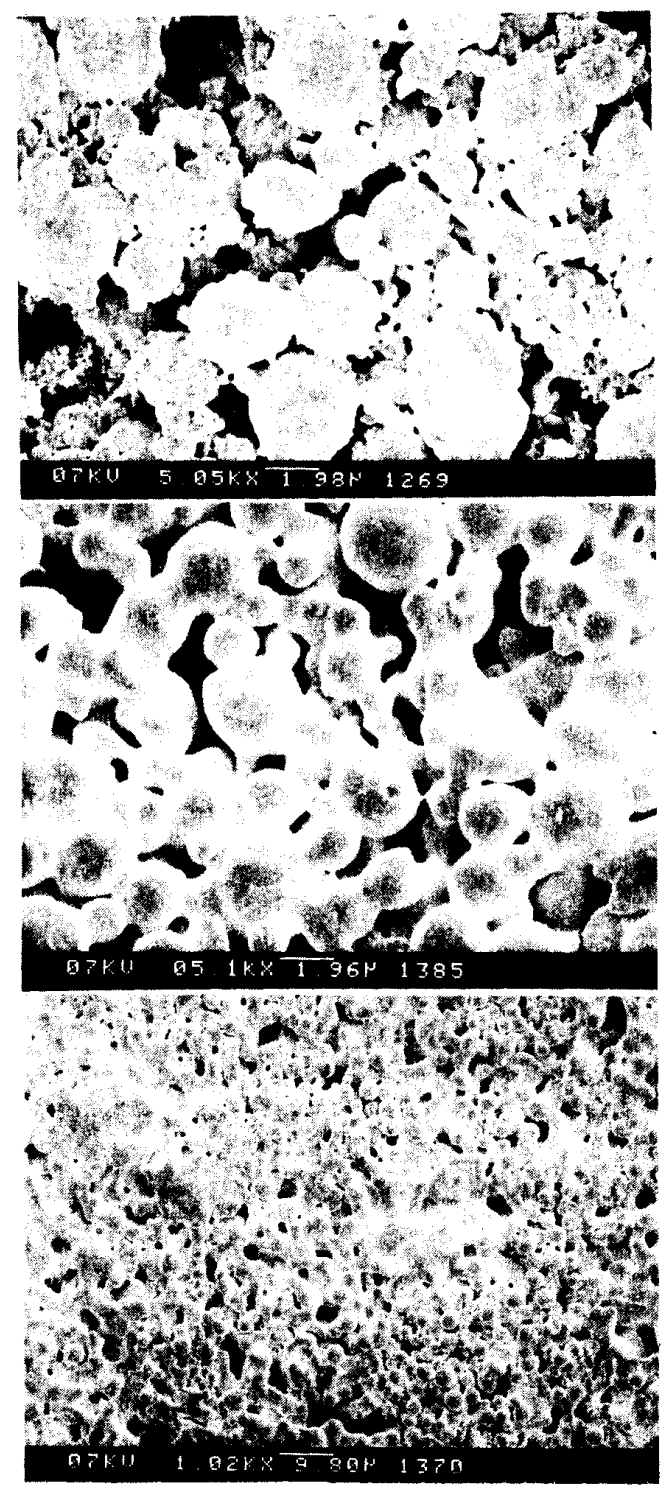

(a)

(c)

Fig.2. Microstructure of the gold thick-film pastes sintered at different temperatures. (a) $500{ }^{\circ} \mathrm{C}$. (b) $600{ }^{\circ} \mathrm{C}$, (c) $700{ }^{\circ} \mathrm{C}$. 
respectively. The pastes have been put into HF-etch for one hour after sintering in order to etch the glassy compound. From these results, the temperature of $600^{\circ} \mathrm{C}$ was found to be optimal. At $700{ }^{\circ} \mathrm{C}$ some parts of the particles are melted completely and forms a continuous area, whereas $500{ }^{\circ} \mathrm{C}$ is too low for the particles to connect. The nitrogen ambient is necessary because it was found that some oxides were formed during the sintering in the presence of oxygen and can hardly be etched later on.

\section{The sacrificial layer}

The selection of the sacrificial layer is another important step, because it has to meet some critical requirements in the later processes. The main requirements are: a) able to withstand high temperature $\left(600{ }^{\circ} \mathrm{C}\right)$ without reacting with gold, b) provide a good protection against HF etching when removing the glassy compound of the thick-film paste, c) be easily removed after formation of the porous actuator. Materials which meet all the requirements are rarely found. For this reason, the sandwich structure of $\mathrm{SiO}_{2} / \mathrm{Si} / \mathrm{SiO}_{2}$ was chosen. The advantages of silicon are the capability of withstanding HF etching and the ease of being etched, unfortunately, it will react with gold above $375{ }^{\circ} \mathrm{C}$. The silicon dioxide cannot withstand $\mathrm{HF}$ etching but it can withstand $600{ }^{\circ} \mathrm{C}$ without reacting with gold and can also easily be etched. The sandwich structure combines both advantages of the two materials. The final results proved that this structure was successful.

\section{Results and discussions}

The device was tested by performing an acid-to-base titration. The same titration using an original prototype device was also performed for comparison. In order to check the reduction of the delay time, the titrations occurring at both the actuator and at the gate of the ISFET were determined. The former was done by a classical technique known as chronopotentiometry. The potential of the actuator versus S.C.E. was continuously recorded during titration and the equivalence point was determined from its first derivative. The latter was done in a similar way, the only difference is that the output of the ISFET is recorded instead of the potential of the actuator. The measurement set-up has been described in a previous paper [4]. The registration curves and the first derivatives are shown in Fig.3a-b. The equivalence points reached at the actuator and the gate of the ISFET can be easily observed from their first derivatives. The delay time can then be directly calculated. In the case of a prototype device with planar actuator around the gate, it

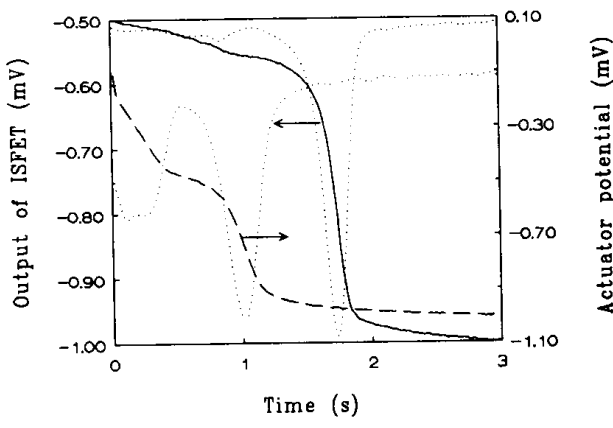

(a)

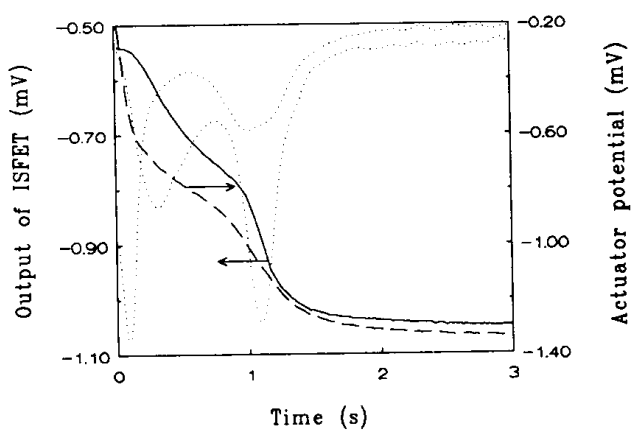

(b)

Fig.3. Registration curves of an acid-base titration and the potential of the actuator, dotted lines show the first derivatives. [HAc] $=2.0 \mathrm{mM},[\mathrm{KNO}]=,0.1 \mathrm{M}$. (a) Device with planar actuator. (b) Device with porous actuator.

can be seen from Fig.3a that when the time of titration at the actuator is one second, the time of titration at the gate of the ISFET is 1.74 seconds. Thus, the delay time is 0.74 seconds. For the device with a porous actuator, the time of titration at the gate of the ISFET was only 0.1 second more than the same titration time of one second at the actuator (Fig.3b). The delay time is considerably shortened compared with that of the prototype device.

A disadvantage of the new device, as found in the experiments, is that the sharpness of the equivalence point in the registration curve is decreased due to an increased output of the ISFET before titration (Fig.3b). The reason is that the porous actuator is in between the measuring loop, the titration curve is deformed by the additional polarization potential of the porous actuator. For a relatively long time of titration or high current density in a high concentration of species, the equivalence point becomes difficult to be detected. In the experiments, the titration times of the new device were found to be from 0.1 to ca. 4.0 seconds, which are shorter than those of the original device. The corresponding concentrations of the species range from 0.5 to ca. $15 \mathrm{mM}$. The titration times as a function of the concentration for acetic acid titration are shown in Fig.4. An approximate 


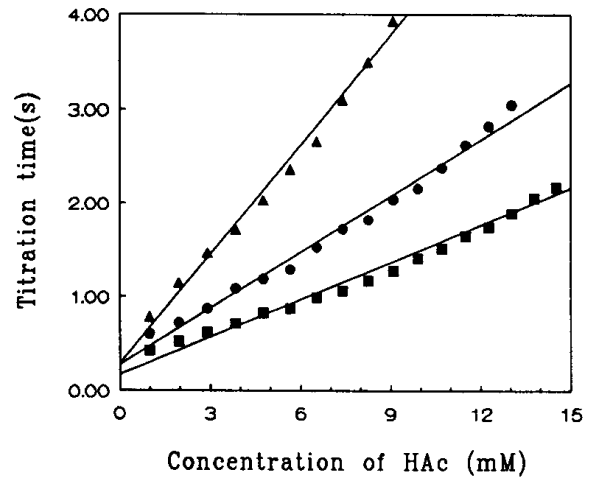

Fig.4. Titration time as a function of the concentration of the acetic acid. Applied currents are: $\triangle-20 \mu \mathrm{A},-30 \mu \mathrm{A}$, - $-40 \mu \mathrm{A}$

linear relation was found between the titration time and the concentration of the species. This is different from the prototype device with a flat actuator for which a linear relation exists between the square root of titration time and the concentration of the species. The reason is that the titrants are not only generated at the surface of the actuator, but also inside the pores and at a much smaller distance from the gate. The mass transport of free diffusion exerts less influence upon the titration time.

\section{Conclusions}

An ISFET-based coulometric sensor-actuator system with a porous noble metal actuator has been successfully developed. The experimental results showed that the delay in response of the system was considerably reduced. However, the polarization potential of the actuator influences the output of the ISFET and distorts the titration curve, which shortens the upper limit of the titration time to ca. 4 seconds.

\section{$\underline{\text { Acknowledgement }}$}

The technical assistance of J. Bomer is greatly appreciated.

\section{References}

1 W. Olthuis, B.H. v.d. Schoot, F. Chavez and P. Bergveld, A Dipstick Sensor for Coulometric Acid-base Titrations, Sensors and Actuators, 17 (1989) 279-283.

2 B.H. v.d. Schoot and P. Bergveld, ISFET-based Enzyme Sensors, Biosensors, 3 (1988) 161-186.

3 B.H. v.d. Schoot and P. Bergveld, Coulometric Sensors,
The Application of a Sensor-actuator System for Long-term Use in Chemical Sensing, Sensors and Actuator 13 (1988) 251-262.

4 W. Olthuis, J. Luo, B.H. v.d. Schoot, P. Bergveld, M. Bos, and W.E. v.d. Linden, Modelling of Non-steady State Concentration Profiles at ISFET-based Coulometric Sensor-actuator Systems, Anal. Chim. Acta, 229 (1990) 71.

5 P. Bergveld and A. Sibbald, Comprehensive Analytical Chemistry, Vol.XXIII, Elsevier Amsterdam, 1988. 